\title{
The Relationship of Functional Status of Cortisol, Testosterone, and Parameters of Metabolic Syndrome in Male Schizophrenics
}

\author{
Yi Han, ${ }^{1}$ Haifeng $\mathrm{Ji}^{2}{ }^{2}$ Li Liu, ${ }^{2}$ Yuncheng Zhu $\mathbb{D}^{2,3}$ and Xixi Jiang $\mathbb{D}^{2}$ \\ ${ }^{1}$ Navy Characteristic Medical Center of PLA, Shanghai 200052, China \\ ${ }^{2}$ Shanghai Changning Mental Health Center (Affiliated to East China Normal University), Shanghai 200335, China \\ ${ }^{3}$ Shanghai Mental Health Center, Shanghai Jiao Tong University School of Medicine, Shanghai 200030, China
}

Correspondence should be addressed to Yuncheng Zhu; hellfiregenius@163.com and Xixi Jiang; 226334295@qq.com

Received 19 August 2020; Revised 6 October 2020; Accepted 8 October 2020; Published 23 October 2020

Academic Editor: Dorota Formanowicz

Copyright ( 2020 Yi Han et al. This is an open access article distributed under the Creative Commons Attribution License, which permits unrestricted use, distribution, and reproduction in any medium, provided the original work is properly cited.

Background. The cross-sectional study is aimed at investigating the relationship between cortisol, testosterone, and metabolic characteristics among male schizophrenics. Methods. 174 patients were grouped based on their risk of metabolic syndrome (MetS) into the non-MetS, high-risk-MetS (HR-MetS), or MetS groups. Metabolic indices (body mass index (BMI), mean arterial pressure (MAP), cholesterol, triglyceride, and fasting blood glucose (FBG)) were associated with cortisol and testosterone levels using correlation analysis. Multiple linear regression analysis was used to associate the correlations between the WHO Quality of Life-BREF (WHOQOL-BREF) score and the five metabolic indices. Results. The WHOQOL-BREF score for the non-MetS group significantly differed from the scores of the HR-MetS and MetS groups. The triglyceride level was positively correlated with the cortisol level, while all five metabolic indices were negatively correlated with testosterone level. Stepwise regression analysis produced a model predicting WHOQOL-BREF scores with four variables including MAP, intelligence quotient (IQ), FBG, and age. The correlation analysis then showed that there was a weak linear correlation between the testosterone level and all five metabolic indices. Conclusions. Among the five metabolic indices, the risks of hypertension and hyperglycemia are correlated with the quality of life in male schizophrenics rather than those of obesity or hyperlipidemia.

\section{Background}

Metabolic syndrome (MetS) has a higher comorbidity with mental illness [1], which is a major reason that the life expectancy of patients with schizophrenia is much lower than that of healthy people [2]. MetS is clinically similar to Cushing's disease in many aspects that may be closely associated with adrenal steroids [3], in which the cortisol level may be positively correlated with MetS [4]. Moreover, androgen promotes the balance of positive nitrogen, which has a positive biological regulating effect on blood glucose and lipid. Testosterone level is negatively associated with MetS in older men [5]. Therefore, males are less likely to gain weight and suffer from metabolic diseases in their early adulthood [6].

A previous report of the cortisol-todehydroepiandrosterone sulfate $(\mathrm{C} / \mathrm{D})$ ratio might predict health levels of patients [7]. Meanwhile, Smith et al. [8] drew a different conclusion that the increased cortisol-to- testosterone $(\mathrm{C} / \mathrm{T})$ ratio is positively correlated with metabolic factors such as coronary heart disease and insulin resistance syndrome, and the ratio is negatively related with chronic stress [9]. However, Gallagher et al. [10] found significant increases of cortisol and dehydroepiandrosterone sulfate (DHEA-S) in the chronic course of schizophrenia. Only when DHEA-S is transformed into dehydroepiandrosterone by the action of sulfatase in peripheral tissues can it be further transformed into testosterone to produce biological effects [11]. About $90 \%$ of the blood circulation of DHEA-S comes from the adrenal cortical reticulum, and the serum concentration is mostly used to evaluate the situation of suspected adrenal androgen overproduction. DHEA$\mathrm{S}$ can be converted to either androgens or estrogens impacted by gender differences. So, to focus on downstream testosterone may be more accurate than DHEA-S by further controlling the gender variable. As far as we know, there is no specific study on the downstream $\mathrm{C} / \mathrm{T}$ ratio and MetS 
correlation among male patients with schizophrenia in China. We tested the relationship between $\mathrm{C} / \mathrm{T}$ ratio and risk of MetS of schizophrenia, to explore the role of $\mathrm{C} / \mathrm{T}$ ratio and to better assess disease risk prediction.

Additionally, compared with demography and symptomatology of schizophrenia, the treatment strategies [12] and quality of life (QOL) for patients [13] are of more urgent concern for the therapeutic alliance [14]. The investigation of the above issues must be based on patients' good treatment compliance, life satisfaction, and happiness [15]. MetS is a cluster of metabolic disorders, in which many metabolic components in the body are abnormal. It is considered a high-risk factor for diabetes, cardiovascular and cerebrovascular diseases, weight gain, and impaired glucose tolerance, all of which contribute to QOL declines in schizophrenics [1]. Metabolic diseases are more common in psychiatric patients due to disturbances of glucose and lipid metabolism related to antipsychotic drugs [16], inadequate individual nutrition education [17], and self-neglect [18]. Based on the above considerations, we aimed to investigate the relationship between the MetS and $\mathrm{C} / \mathrm{T}$ ratio as the primary objective and MetS and QOL as the secondary.

\section{Methods}

2.1. Participants and Procedure. Our participants were recruited through the outpatient service system of the Shanghai Changning Center for Disease Control, from 01/01/2015 to $06 / 30 / 2016$. The mental health services of the district cover nine jurisdictions and one town. The diagnosis of schizophrenia was ascertained according to the International Statistical Classification of Diseases and Related Health Problems, Tenth Revision (ICD-10). Inclusion criteria were as follows: male, Chinese Han ethnicity, aged 18 to 60 years, Wechsler Adult Intelligence Scale - Fourth Edition (WAIS - IV) $\geq 70$, and steady long-term use (more than a year) of one antipsychotic drug. Exclusion criteria were as follows: metabolic disorder(s) on medication, alcohol or drug abuse, primary neuroendocrine diseases (thyroid axis, adrenal axis, gonad axis, pituitary tumor, etc.), severe physical disease(s) comorbidity, and having taken drugs for other disease(s) within two weeks.

The enrolled patients were divided according to the Chinese Diabetes Society criteria guideline for MetS as follows [19]: (1) overweight and/or obese: body mass index (BMI) $\geq 25.0 \mathrm{~kg} / \mathrm{m}^{2}$; (2) hyperglycemia: fasting blood glucose (FBG ) $\geq 6.1 \mathrm{mmol} / \mathrm{l}$ and/or 2 - hour postprandial blood glucose $\geq$ $7.8 \mathrm{mmol} / \mathrm{l}$, and/or having been diagnosed with diabetes or on medication; (3) hypertension: blood pressure $\geq 140 / 90$ $\mathrm{mmHg}$ and/or having been diagnosed with hypertension or on medication; and (4) dyslipidemia: fasting blood triglycerides $\geq 1.7 \mathrm{mmol} / \mathrm{l}$ and/or fasting blood high density lipoprotein (male) $<0.9 \mathrm{mmol} / \mathrm{l}$. The outpatient service system followed the database of patients with single medication, among which 250 patients met the requirements and agreed to participate. There were 32 cases $(12.8 \%)$ on medication for at least one metabolic disorder: twentyseven cases $(10.8 \%)$ had been diagnosed as no less than one of the metabolic disorders and five cases $(2.0 \%)$ as MetS (three or more metabolic disorders). We then analyzed the remaining 174 cases (70\%) who met the above criteria enrolled in the study and completed the investigation (see Figure 1 for a flow diagram of sample selection).

Our study was approved on 01/01/2015 by the Institutional Ethical Committee for clinical research of Shanghai Changning Mental Health Center, Shanghai, China. Written informed consent was provided according to the Declaration of Helsinki and obtained from participants or their next of kin.

\subsection{Measures}

2.2.1. Wechsler Adult Intelligence Scale-Fourth Edition (WAIS-IV). The WAIS-IV can be used to assess the intelligence quotient (IQ) level for schizophrenia [20]. With an average IQ of 100, the higher the total IQ score, the higher the overall cognitive ability of the individual will be when compared with the same group of people.

2.2.2. Positive and Negative Syndrome Scale (PANSS). The Chinese Mandarin version of the PANSS [21] has been shown to be a reliable and valid instrument for the assessment of the severity of psychopathology in schizophrenics. The scale consists of 30 items, each rated using a 7-point scale. We recorded patients' total PANSS and subscales: positive symptoms, negative symptoms, and general psychopathology scores.

2.2.3. Body Mass Index (BMI) and Mean Arterial Pressure (MAP). The calculation of BMI is the weight $(\mathrm{kg})$ of the individual divided by the squared height $\left(\mathrm{m}^{2}\right)$. It is the current international standard to measure the body fat and fitness level of humans. BMI is an objective and reliable value when comparing and analyzing individuals' weight on different heights [22]. In China, the normal range is from 18.5 to $25.0 \mathrm{~kg} / \mathrm{m}^{2}$. Underweight is below the normal value while overweight is above it.

MAP is the average arterial blood pressure during a cardiac cycle. The mean arterial pressure of normal adults ranged from 70 to $105 \mathrm{mmHg}$ (MAP $=1 / 3$ systolic blood pressure $+2 / 3$ diastolic blood pressure).

2.2.4. WHO Quality of Life-BREF (WHOQOL-BREF). WHOQOL-BREF has good reliability, validity, and international comparability, which can be used for the assessment of QOL of MetS in schizophrenia [23]. The scale consists of 26 items, each rated using a 5-point scale including physiological, psychological, social, and environmental fields. The $3 \mathrm{rd}$, 4th, and 26th items were adjusted by reverse scoring. Scores range from 26 to 130, with higher scores indicating better QOL.

2.2.5. Haemoconcentration of Cortisol, Testosterone, FBG, Triglycerides, and Cholesterol. Nutrition consultation according to individuals' base levels was conducted to provide professional energy intake recommendations. Blood samples were then collected after patients had been conforming to 


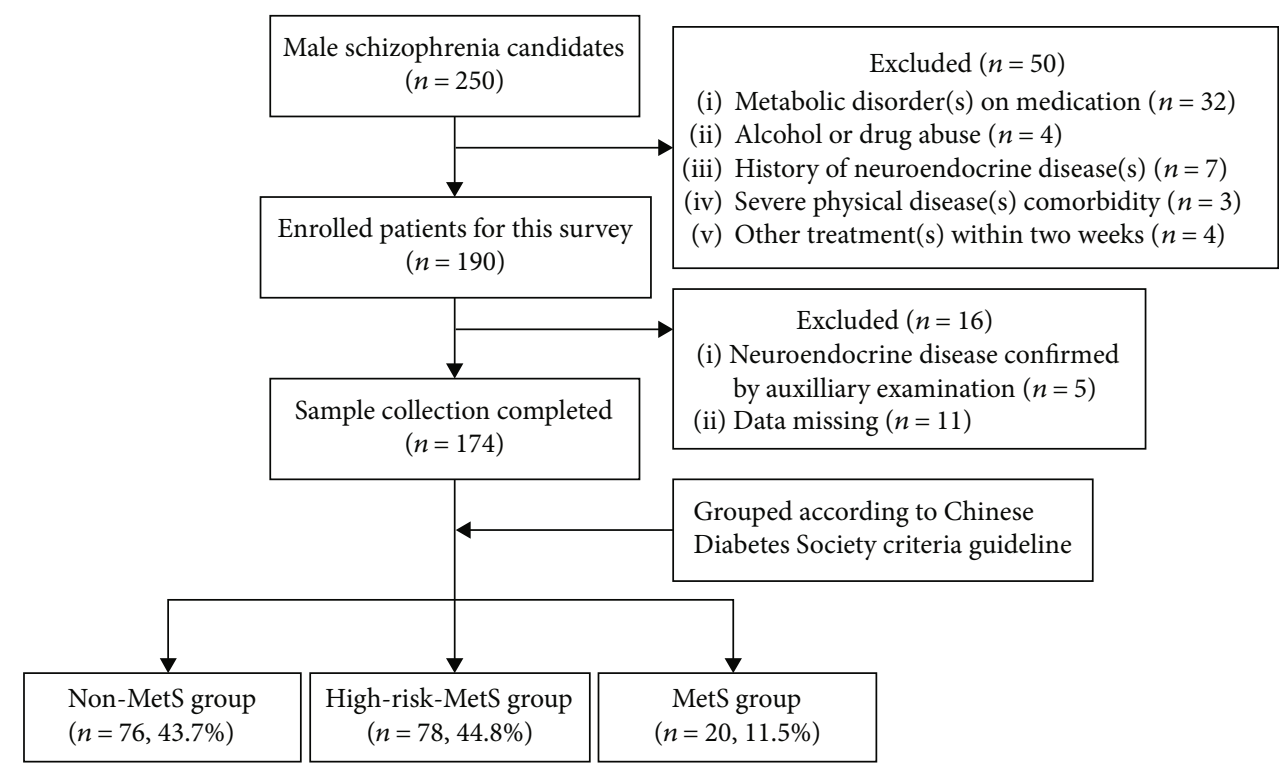

FIGURE 1: Flowchart of the screening process and data classification. We enrolled 174 male schizophrenics for each group from 250 candidates over those exclusion criteria. These cases were grouped by disease severity of MetS (76 cases in non-MetS group, 78 cases in high-risk-MetS group, with one or two of the above metabolic disorders, and 20 cases in MetS group, with three or more metabolic disorders).

the recommendations for at least one month. Fasting blood test was taken at 7 a.m. Electrochemical luminescence immunoassay was performed using Roche Modular E170 (cortisol and testosterone) [24] and Modular P800 (FBG, triglycerides, and cholesterol) [25] automatic electrochemiluminescence immunoassay system, provided by Shanghai Lanwei Clinical Testing.

2.2.6. Statistical Analysis. All statistical computations were performed using SPSS 22.0. All measurement values were confirmed for normality by means of the KolmogorovSmirnov test and homogeneity test of variance. Normal distribution data were represented as mean $( \pm S D)$. Differences in age, course of disease, IQ, WHOQOL-BREF, cortisol, testosterone, and $\mathrm{C} / \mathrm{T}$ were analyzed using analysis of variance. Metabolic indices (BMI, MAP, FBG, triglyceride, and cholesterol) were associated with cortisol and testosterone levels using Pearson's correlations and hierarchical multiple linear regression analysis. Stepwise multiple linear regression analysis was carried out to examine the relationships between the WHOQOL-BREF score and the five metabolic indices, cortisol, testosterone, age, course of disease, onset age, and IQ. The probabilities for entry and removal were 0.1 and 0.15 , respectively.

\section{Results}

3.1. Demographic Information and Clinical Features. For the 174 patients, the average age was $48.4( \pm 10.0)$ years, course of disease $23.5( \pm 11.4)$ years, and onset age $24.9( \pm 8.0)$ years. The most commonly used medications were as follows: 40 cases of olanzapine (23.0\%), 29 of clozapine (16.7\%), 28 of risperidone $(16.1 \%), 15$ of quetiapine $(8.6 \%)$, and 14 of chlorpromazine $(8.0 \%)$.
Table 1 presents the descriptive statistics for the different metabolic groups. There were no significant between-group differences in age, course of disease, onset age, IQ, and total and subscale PANSS scores $(p>0.05)$. WHOQOL-BREF scores showed significant differences between the groups $(p<0.01)$, and further post hoc LSD comparisons between the non-MetS group and the other two groups were statistically significant $(p=0.009 / 0.002)$, while the difference between the high-risk-MetS (HR-MetS) group and the MetS group was not $(p=0.153)$.

3.2. Metabolic Indices. Table 2 presents the statistics for the metabolic indices among the different patient groups. BMI, MAP, FBG, triglyceride, cholesterol, and testosterone levels and $\mathrm{C} / \mathrm{T}$ ratio showed statistical differences $(p<0.01)$ between groups. There was no statistically difference in the cortisol level among the three groups $(p=0.110)$.

3.3. Correlations between Predictors and Metabolic Risk Factors in Male Schizophrenics. In the correlation analysis between the five metabolic indices and three predictors (cortisol, testosterone, and $\mathrm{C} / \mathrm{T}$ ratio), the triglyceride level was positively correlated with the cortisol level $(r=0.221, p=0.003)$, while BMI, MAP, cholesterol, and FBG levels had no correlations with cortisol level $(p>0.05)$. All five metabolic indices were negatively correlated with testosterone level $(r[-0.343,-0.211], p<0.05)$ and positively correlated with $\mathrm{C} / \mathrm{T}$ ratio $(r[0.149,0.211], p<0.05)$, as shown in Table 3 .

To observe the interaction effect, the hierarchical multiple linear regression models of the five regression analyses were employed, shown in Table 4. The multiple linear relationships of all five metabolic indices and the two predictors were statistically significant $(p<0.05)$. The triglyceride level was positively correlated with the cortisol level $(p<0.05)$, and all five 
TABLE 1: Statistics of demographic information and clinical features of male schizophrenics across different metabolic risk groups.

\begin{tabular}{|c|c|c|c|c|c|}
\hline & Non-MetS $(n=76)$ & HR-MetS $(n=78)$ & MetS $(n=20)$ & $F$ & $p$ value \\
\hline Age (y) & $49.6 \pm 9.3$ & $47.8 \pm 10.2$ & $46.0 \pm 11.1$ & 1.318 & 0.27 \\
\hline Course of disease $(y)$ & $24.8 \pm 10.1$ & $23.3 \pm 13.0$ & $19.6 \pm 9.2$ & 1.691 & 0.19 \\
\hline Onset age (y) & $24.8 \pm 7.9$ & $24.5 \pm 8.1$ & $26.4 \pm 8.3$ & 0.452 & 0.64 \\
\hline IQ & $82.6 \pm 9.9$ & $82.5 \pm 8.2$ & $84.6 \pm 10.7$ & 0.394 & 0.68 \\
\hline Total PANSS & $87.3 \pm 14.8$ & $85.7 \pm 11.6$ & $83.6 \pm 7.1$ & 0.776 & 0.46 \\
\hline Positive symptoms & $19.7 \pm 6.3$ & $20.0 \pm 5.7$ & $17.7 \pm 4.7$ & 1.095 & 0.34 \\
\hline Negative symptoms & $18.2 \pm 4.7$ & $17.5 \pm 4.9$ & $18.6 \pm 5.3$ & 0.653 & 0.52 \\
\hline General psychopathology & $38.0 \pm 5.3$ & $37.6 \pm 5.4$ & $36.5 \pm 3.5$ & 1.482 & 0.23 \\
\hline WHOQOL-BREF & $82.4 \pm 8.4$ & $78.3 \pm 10.0$ & $74.9 \pm 11.2$ & 5.903 & $<0.01$ \\
\hline
\end{tabular}

IQ: intelligence quotient; WHOQOL-BREF: WHO Quality of Life-BREF; PANSS: Positive and Negative Syndrome Scale.

TABLE 2: Metabolic indices of male schizophrenics across different metabolic risk groups.

\begin{tabular}{|c|c|c|c|c|c|}
\hline & Non-MetS $(n=76)$ & HR-MetS $(n=78)$ & MetS $(n=20)$ & $F$ & $p$ value \\
\hline BMI $\left(\mathrm{kg} / \mathrm{m}^{2}\right)$ & $21.4 \pm 2.4$ & $24.3 \pm 4.1$ & $28.2 \pm 2.7$ & 24.755 & $<0.01$ \\
\hline MAP (mmHg) & $87.9 \pm 8.8$ & $98.5 \pm 13.5$ & $107.5 \pm 8.3$ & 22.713 & $<0.01$ \\
\hline $\mathrm{FBG}(\mathrm{mmol} / \mathrm{l})$ & $4.6 \pm 0.4$ & $5.0 \pm 0.8$ & $5.8 \pm 1.0$ & 15.776 & $<0.01$ \\
\hline Triglyceride (mmol/l) & $1.1 \pm 0.4$ & $1.5 \pm 0.6$ & $2.5 \pm 0.4$ & 41.657 & $<0.01$ \\
\hline Cholesterol (mmol/l) & $3.9 \pm 0.9$ & $4.2 \pm 0.7$ & $4.6 \pm 0.8$ & 5.129 & $<0.01$ \\
\hline Cortisol (nmol/l) & $450.9 \pm 128.6$ & $489.6 \pm 157.7$ & $511.9 \pm 102.8$ & 2.245 & 0.11 \\
\hline Testosterone (nmol/l) & $18.8 \pm 7.4$ & $15.5 \pm 7.4$ & $12.3 \pm 3.1$ & 8.379 & $<0.01$ \\
\hline $\mathrm{C} / \mathrm{T}$ ratio & $28.0 \pm 15.0$ & $40.5 \pm 25.5$ & $45.7 \pm 19.7$ & 9.623 & $<0.01$ \\
\hline
\end{tabular}

C/T ratio: cortisol-to-testosterone ratio; BMI: body mass index; MAP: mean arterial pressure; FBG: fasting blood glucose.

TABLE 3: Linear correlation analysis between predictors and metabolic risk factors in male schizophrenics.

\begin{tabular}{|c|c|c|c|c|c|}
\hline & BMI $\left(\mathrm{kg} / \mathrm{m}^{2}\right)$ & MAP (mmHg) & Cholesterol $(\mathrm{mmol} / \mathrm{l})$ & Triglyceride $(\mathrm{mmol} / \mathrm{l})$ & $\overline{\text { FBG }(\mathrm{mmol} / \mathrm{l})}$ \\
\hline Cortisol (nmol/l) $(r)$ & -0.061 & 0.065 & 0.042 & 0.221 & 0.056 \\
\hline$F$ & 0.637 & 0.730 & 0.304 & 8.792 & 0.547 \\
\hline$p$ & 0.426 & 0.394 & 0.582 & 0.003 & 0.460 \\
\hline Testosterone $(\mathrm{nmol} / \mathrm{l})(r)$ & -0.343 & -0.240 & -0.211 & -0.327 & -0.211 \\
\hline$F$ & 22.999 & 10.522 & 8.019 & 20.646 & 7.992 \\
\hline$p$ & 0.000 & 0.001 & 0.005 & 0.000 & 0.005 \\
\hline $\mathrm{C} / \mathrm{T}$ ratio $(r)$ & 0.165 & 0.211 & 0.149 & 0.199 & 0.152 \\
\hline$F$ & 4.841 & 8.016 & 3.903 & 7.127 & 4.092 \\
\hline$p$ & 0.029 & 0.005 & 0.050 & 0.008 & 0.045 \\
\hline
\end{tabular}

C/T ratio: ratio of cortisol to testosterone; BMI: body mass index; MAP: mean arterial pressure; FBG: fasting blood glucose.

metabolic indices were negatively correlated with testosterone level $(p<0.05)$. These results were consistent with those using Pearson's correlation analysis. Furthermore, the absolute $r$ values in Table 4 were higher than those of all three predictors in Table 3, especially compared to those of testosterone.

3.4. Predictors of WHOQOL-BREF. In this study, we attempted to associate WHOQOL-BREF scores with BMI,
MAP, FBG, triglyceride, cholesterol, cortisol, testosterone, age, course of disease, onset age, and IQ through stepwise multiple linear regression. The final regression model, which comprise the predictors MAP, IQ, FBG, and age $\left(F_{(3,174)}=4.618\right.$, $p=0.001, r^{2}=0.099$, and $\left.\Delta r^{2}=0.077\right)$, is shown in Table 5 . The regression equation was obtained as follows: $y=123.705$ $-0.131 x_{1}-0.204 x_{2}-1.78 x_{3}-0.123 x_{4}, x_{1}=\operatorname{MAP}(\mathrm{mmHg})$ , $x_{2}=\mathrm{IQ}, x_{3}=\mathrm{FBG}(\mathrm{mmol} / \mathrm{l}), x_{4}=\operatorname{age}(\mathrm{y})$. 
TABLE 4: Hierarchical multiple linear regression analysis of cortisol and testosterone levels for each metabolic parameters.

\begin{tabular}{|c|c|c|c|c|c|c|c|c|}
\hline Model & $\beta^{\mathrm{a}}$ & S.E. & $\beta^{\mathrm{b}}$ & $t$ & $p$ & $r$ & $r^{2}$ & $\Delta r^{2}$ \\
\hline BMI & & & & & & -0.369 & 0.136 & 0.126 \\
\hline Constant & 28.403 & 1.266 & & 22.443 & $<0.001$ & & & \\
\hline Cortisol & -0.003 & 0.002 & -0.123 & 1.705 & 0.090 & & & \\
\hline Testosterone & -0.197 & 0.038 & -0.369 & 5.113 & $<0.001$ & & & \\
\hline MAP & & & & & & -0.248 & 0.062 & 0.051 \\
\hline Constant & 100.977 & 4.355 & & 23.187 & $<0.001$ & & & \\
\hline Cortisol & 0.002 & 0.007 & 0.024 & 0.319 & 0.750 & & & \\
\hline Testosterone & -0.429 & 0.132 & -0.243 & 3.237 & 0.001 & & & \\
\hline FBG & & & & & & -0.214 & 0.046 & 0.035 \\
\hline Constant & 5.238 & 0.264 & & 19.874 & $<0.001$ & & & \\
\hline Cortisol & 0.000 & 0.000 & 0.021 & 0.277 & 0.782 & & & \\
\hline Testosterone & -0.022 & 0.008 & -0.209 & 2.762 & 0.006 & & & \\
\hline Triglyceride & & & & & & -0.368 & 0.135 & 0.125 \\
\hline Constant & 1.514 & 0.204 & & 7.432 & $<0.001$ & & & \\
\hline Cortisol & 0.001 & 0.000 & 0.170 & 2.358 & 0.019 & & & \\
\hline Testosterone & -0.026 & 0.006 & -0.299 & -4.142 & $<0.001$ & & & \\
\hline Cholesterol & & & & & & -0.213 & 0.045 & 0.034 \\
\hline Constant & 4.480 & 0.284 & & 15.797 & $<0.001$ & & & \\
\hline Cortisol & 0.000 & 0.000 & 0.006 & 0.082 & 0.934 & & & \\
\hline Testosterone & -0.024 & 0.009 & -0.212 & -2.798 & 0.006 & & & \\
\hline
\end{tabular}

${ }^{\mathrm{ab}}$ Unstandardized coefficients. Standardized coefficients. BMI: body mass Index; MAP: mean arterial pressure; FBG: fasting blood glucose.

TABLE 5: Results of stepwise multiple linear regression analysis for WHOQOL-BREF in male schizophrenics.

\begin{tabular}{lccccccc}
\hline Model & $\beta^{\mathrm{a}}$ & S.E. & $\beta^{\mathrm{b}}$ & $t$ & $p$ & $r$ & $r^{2}$ \\
\hline Constant & 123.705 & 10.409 & & 11.884 & $<0.001$ & -0.314 & 0.099 \\
MAP & -0.131 & 0.045 & -0.174 & -2.313 & 0.022 & 0.077 \\
IQ & -0.204 & 0.078 & -0.194 & -2.261 & 0.010 & \\
FBG & -1.780 & 0.934 & -0.142 & -1.906 & 0.058 & \\
Age & -0.123 & 0.072 & -0.126 & -1.703 & 0.090 & \\
\hline
\end{tabular}

${ }^{\mathrm{a}}$ Unstandardized coefficients. ${ }^{\mathrm{b}}$ Standardized coefficients. MAP: mean arterial pressure; FBG: fasting blood glucose.

The above regression equation showed that MAP, IQ, FBG, and age were negatively correlated with WHOQOLBREF scores.

\section{Discussion}

Under the modern medical system, MetS is still a serious potential risk, increasing incidences of cardiovascular and cerebrovascular diseases. In the present study, we grouped schizophrenic patients according to risk of metabolic abnormalities and examined differences in age, course of disease, and IQ. We used BMI instead of waist circumference for the metabolic index as the most significant predictors of MetS for both men and women in China [26]. The study supports that any abnormality of one metabolic index can be accompanied by changes in other metabolic indices, thus aggravating the severity of disease by increasing the risk of MetS [27]. Of concern is that $32.3 \%$ of the total sample $(76 / 235)$ in our district are diagnosed as MetS and $35.3 \%$
(83/235) suffer from at least one metabolic disorder. The overall time point risk rate reaches up to $67.7 \%$. This data exceeds the overall MetS rate among male adults in China (31.0\%) [28]. Schizophrenia runs a high risk of MetS comorbidity [13]. Our data also showed that the mean age of highrisk MetS in schizophrenics, 48, is younger than the 50-yearold warning line of male MetS in Shanghai [29]. The metabolic risk brought by long-term maintenance treatment must be considered in advance before determining treatment options for patients in the acute stage, rather than merely pursuing short-term treatment effects of medications [30].

The endocrine changes of adrenal and sex steroids may improve cognition in schizophrenia $[31,32]$. However, testosterone secretion in males goes through a process of natural decline with the recession of testicular function. Therefore, our study sample only included male patients who were younger than 60 years old, so as to avoid male menopause with physiological "inflexion point" of testosterone $[33,34]$. We excluded female patients because of physiological low 
levels of testosterone and hyperandrogenemia of polycystic ovarian syndrome which may interfere with the results of MetS. Under this condition, the occurrence of metabolic abnormalities is proportional to the $\mathrm{C} / \mathrm{T}$ ratio and inversely proportional to the testosterone level, consistent with our expectation.

The lack of between-group difference in the cortisol level does not completely negate its predictive significance. Numerous studies have shown that elevated cortisol [35] and decreased testosterone levels [36] can be associated with MetS. Nevertheless, in our study on male patients with schizophrenia, testosterone concentration was found to have a protective effect on five metabolic indices, while cortisol concentration has a facilitation effect on an elevated triglyceride level, consistent with previous studies. For considering the potential interaction effect between the cortisol and testosterone levels, regression analyses showed that the correlation coefficients involving these two predictors were the highest among the regression models. However, the relationship between the C/T ratio and schizophrenia is complicated, relating to the course of disease, age, and duration of antipsychotic medication [31]. It is also possible that the dosage of antipsychotic drugs, which was not recorded in the present study, has an impact. For example, the commonly used low doses of quetiapine and olanzapine, as well as most atypical antipsychotic drugs, reduce cortisol levels in patients with schizophrenia $[37,38]$. Obviously, these results are not satisfactory if they require patients with schizophrenia to stop taking medications, which has never happened in practice. It deserves scientific investigation because the predictiveness of the $\mathrm{C} / \mathrm{T}$ ratio and its application in schizophrenia have not yet been considered in clinical assessment.

Another aim of the study is to observe whether the QOL of patients continuously decrease with increases in metabolic abnormalities. Results indicate that as long as one metabolic abnormality occurs, the QOL of patients decreases significantly, while the downtrend stops at the stage of HR-MetS and MetS. As a result, any metabolic abnormality is a risk factor for patients' QOL as a primary prevention, and it should be of high concern in tertiary prevention. In a meta-analysis, Vancampfort et al. [39] showed that the odds ratios (OR) of metabolic syndrome were induced by antipsychotics: clozapine (7.81), olanzapine (5.87), quetiapine (5.14), classical antipsychotics (4.97), risperidone (4.57), sulfenopril (3.86), and aripiprazole (3.25). Although the clinical efficacy of clozapine and olanzapine is satisfactory, the ORs of MetS caused by these two drugs are the highest. Secondly, due to insufficient family and social support and/or self-neglect to a large extent, most of the patients included in this study did not have proper medical treatment, or those metabolic disorders were even found for the first time. We should provide effective nutritional education on weight change and metabolic abnormalities, such as to encourage exercise, diet regulation, and related medical knowledge to help improve their lifestyle. For example, as a standard care, doctors could provide weight loss advice or an individual nutritional education group as a secondary prevention [17]. Finally, there was negative linear correlation between MAP and FBG levels and QOL scores. The treatment of impaired glucose tolerance

and hypertension affects the patients more than obesity and hyperlipidemia. Our results support the finding that strengthening health education for self-management of diabetes and hypertension in the community could improve patients' life satisfaction [40]. Impaired glucose tolerance and hypertension have become serious factors affecting the QOL of schizophrenia [22]. Our findings support that increasing the prevention and control of these two abnormalities will achieve cost-effectiveness for tertiary preventions [41]. Stepwise multiple linear regression analysis showed that low IQ and aging are also important factors for declines in QOL. Due to their high impact across a range of domains, these two factors need to be understood and cared about by the whole society.

\section{Conclusions}

The reason may be that the effect size of schizophrenia on cortical level weighs heavily, leading to incongruency for people without psychosis [10]. Although further correlation analysis showed that cortisol level is positively correlated with triglyceride, the linearly dependent coefficient $(r)$ is weak, and the predicted effect is then limited [7]. We attempted to find the $\mathrm{C} / \mathrm{T}$ ratio as a prediction risk factor on MetS. Although the $\mathrm{C} / \mathrm{T}$ ratio was statistically significant for the prediction, the absolute $r$ values of regression model involving testosterone and cortisol levels $(0.213,0.369)$ were higher than those of the $\mathrm{C} / \mathrm{T}$ ratio $(0.149,0.211)$. This suggests that the testosterone and cortisol levels may predict MetS risk factors better instead of using the $\mathrm{C} / \mathrm{T}$ ratio.

In view of the high rate of MetS in patients with schizophrenia, the study of cortisol and testosterone in the assessment of patients' health status is of great significance [42]. Future studies can be conducted with expanded sample size, as well as independent studies for female subjects, so as to obtain corresponding statistical relationships between genders. Different clinical types and pharmacological effects can be used as parameters for future research.

\section{Abbreviations}

MetS:

$\mathrm{C} / \mathrm{T}$ :

$\mathrm{C} / \mathrm{D}$ :

DHEA-S:

QOL:

ICD-10:

WAIS-IV:

PANSS:

BMI:

MAP:

WHOQOL-BREF:

FBG:

HR-MetS:
Metabolic syndrome

Cortisol-to-testosterone

Cortisol-to-dehydroepiandrosterone sulfate

Dehydroepiandrosterone sulfate Quality of life

the International Statistical Classification of Diseases and Related Health

Problems, Tenth Revision (ICD-10)

Wechsler Adult Intelligence Scale-

Fourth Edition

Positive and Negative Syndrome Scale

Body mass index

Mean arterial pressure

WHO Quality of Life-BREF

Fasting blood-glucose

High-risk metabolic syndrome. 


\section{Data Availability}

The data that support the findings of this study are available from the Shanghai Changning Mental Health Center. Restrictions apply to the availability of these data, which were used under license for this study.

\section{Ethical Approval}

Our study was based on the approval by the Institutional Ethical Committee for clinical research of the Shanghai Changning Mental Health Center, Shanghai, China. Written informed consent was provided according to the Declaration of Helsinki.

\section{Disclosure}

The funding sources had no role in the design of the study; the collection, analysis, and interpretation of the data; and the writing of the manuscript.

\section{Conflicts of Interest}

The authors declare that they have no conflicts of interest.

\section{Authors' Contributions}

YH wrote and revised the manuscript. HJ and LL contributed to data collection. YZ designed the study. XJ collected and analyzed data and wrote the manuscript. All authors read and approved the manuscript.

\section{Acknowledgments}

We would like to thank Lily Tao for English language editing. This work was supported by the Research Project of Shanghai Changning District Science and Technology Commission (CNKW2018Y23), the Research Project of Shanghai Changning Health and Family Planning Commission (20164Y013), and the Innovation Team Project of Shanghai Changning District.

\section{References}

[1] J. Steiner, H. G. Bernstein, K. Schiltz et al., "Immune system and glucose metabolism interaction in schizophrenia: a chicken-egg dilemma," Progress in NeuroPsychopharmacology \& Biological Psychiatry, vol. 48, pp. 287-294, 2014.

[2] T. M. Laursen, "Life expectancy among persons with schizophrenia or bipolar affective disorder," Schizophrenia Research, vol. 131, no. 1-3, pp. 101-104, 2011.

[3] H. P. Gaete, "Hypothalamus-pituitary-adrenal axis, hair cortisol and the metabolic syndrome," Psychiatria Danubina, vol. 27, no. 1, pp. S504-S505, 2015.

[4] M. J. Weigensberg, C. M. Toledo-Corral, and M. I. Goran, "Association between the metabolic syndrome and serum cortisol in overweight Latino youth," The Journal of Clinical Endocrinology and Metabolism, vol. 93, no. 4, pp. 1372-1378, 2008.
[5] M. Maggio, F. Lauretani, G. P. Ceda et al., "Association between hormones and metabolic syndrome in older Italian men," Journal of the American Geriatrics Society, vol. 54, no. 12, pp. 1832-1838, 2006.

[6] G. Corona, M. Monami, G. Rastrelli et al., "Testosterone and metabolic syndrome: a meta-analysis study," The Journal of Sexual Medicine, vol. 8, no. 1, pp. 272-283, 2011.

[7] B. Vuksan-Ćusa, M. Šagud, A. Mihaljević-Peleš, N. Jakšić, and M. Jakovljević, "Metabolic syndrome and cortisol/DHEAS ratio in patients with bipolar disorder and schizophrenia," $P$ sychiatria Danubina, vol. 26, no. 2, pp. 187-189, 2014.

[8] G. D. Smith, Y. Ben-Shlomo, A. Beswick, J. Yarnell, S. Lightman, and P. Elwood, "Cortisol, testosterone, and coronary heart disease prospective evidence from the Caerphilly Study," Circulation, vol. 112, no. 3, pp. 332-340, 2005.

[9] A. Romero-Martinez and L. Moya-Albiol, "The use of testosterone/cortisol ratio in response to acute stress as an indicator of propensity to anger in informal caregivers," Spanish Journal of Psychology, vol. 19, no. 19, p. 62, 2016.

[10] P. Gallagher, S. Watson, M. Smith, A. Young, and I. Ferrier, "Plasma cortisol-dehydroepiandrosterone (DHEA) ratios in schizophrenia and bipolar disorder," Schizophrenia Research, vol. 90, no. 1-3, pp. 258-265, 2007.

[11] W. U. Yuanmin, B. Shao, and G. U. Huilin, "Epidemiologic study on metabolic syndrome among Chinese over 40 of Shanghai Huayang community," Shanghai Medical Journal, vol. 24, no. 4, pp. 195-198, 2001.

[12] M. De Hert, J. Sermon, P. Geerts, K. Vansteelandt, J. Peuskens, and J. Detraux, "The use of continuous treatment versus placebo or intermittent treatment strategies in stabilized patients with schizophrenia: a systematic review and meta-analysis of randomized controlled trials with first- and secondgeneration antipsychotics," CNS Drugs, vol. 29, no. 8, pp. 637-658, 2015.

[13] M. Dauwan, M. J. H. Begemann, S. M. Heringa, and I. E. Sommer, "Exercise improves clinical symptoms, quality of life, global functioning, and depression in schizophrenia: a systematic review and meta-analysis," Schizophrenia Bulletin, vol. 42, no. 3, pp. 588-599, 2016.

[14] Y. Zhu, Z. Wu, O. Sie et al., "Causes of drug discontinuation in patients with major depressive disorder in China," Progress in Neuro-Psychopharmacology and Biological Psychiatry, vol. 96, no. 109755 , p. $109755,2020$.

[15] M. Holubova, J. Prasko, R. Hruby et al., "Coping strategies and quality of life in schizophrenia: cross-sectional study," Neuropsychiatric Disease and Treatment, vol. 11, pp. 3041-3048, 2015.

[16] A. Enez Darcin, S. Yalcin Cavus, N. Dilbaz, H. Kaya, and E. Dogan, "Metabolic syndrome in drug-naïve and drug-free patients with schizophrenia and in their siblings," Schizophrenia Research, vol. 166, no. 1-3, pp. 201-206, 2015.

[17] N. Sugawara, T. Sagae, N. Yasui-Furukori et al., "Effects of nutritional education on weight change and metabolic abnormalities among patients with schizophrenia in Japan: a randomized controlled trial," Journal of Psychiatric Research, vol. 97, pp. 77-83, 2018.

[18] N. Jayatilleke, R. D. Hayes, C. K. Chang, and R. Stewart, "Acute general hospital admissions in people with serious mental illness," Psychological Medicine, vol. 48, no. 16, pp. 2676-2683, 2018.

[19] Y. Lan, Z. Mai, S. Zhou et al., "Prevalence of metabolic syndrome in China: an up-dated cross-sectional study," Plos One, vol. 13, no. 4, p. e0196012, 2018. 
[20] N. M. Michel, J. O. Goldberg, R. W. Heinrichs, A. A. Miles, N. Ammari, and S. McDermid Vaz, "WAIS-IV profile of cognition in schizophrenia," Assessment, vol. 20, no. 4, pp. 462$473,2013$.

[21] B. J. Wu, T. H. Lan, T. M. Hu, S. M. Lee, and J. Y. Liou, "Validation of a five-factor model of a Chinese Mandarin version of the Positive and Negative Syndrome Scale (CMV-PANSS) in a sample of 813 schizophrenia patients," Schizophrenia Research, vol. 169, no. 1-3, pp. 489-490, 2015.

[22] H. Tuzun, S. Aycan, and M. N. Ilhan, "Impact of comorbidity and socioeconomic status on quality of life in patients with chronic diseases who attend primary health care centres," Central European Journal of Public Health, vol. 23, no. 3, pp. 188194, 2015.

[23] C. T. Su, H. S. Ng, A. L. Yang, and C. Y. Lin, "Psychometric evaluation of the Short Form 36 Health Survey (SF-36) and the World Health Organization Quality of Life Scale Brief Version (WHOQOL-BREF) for patients with schizophrenia," Psychological Assessment, vol. 26, no. 3, pp. 980-989, 2014.

[24] C. Bieglmayer, D. W. Chan, L. Sokoll et al., "Multicentre performance evaluation of the E170 module for modular analytics," Clinical Chemistry and Laboratory Medicine, vol. 42, no. 10, pp. 1186-1202, 2004.

[25] G. L. Horowitz, Z. Zaman, N. J. Blanckaert et al., "Modular analytics: a new approach to automation in the clinical laboratory," Journal of Analytical Methods in Chemistry, vol. 2005, 8 pages, 2005.

[26] F. Y. Yang, M. L. Wahlqvist, and M. S. Lee, "Body mass index (BMI) as a major factor in the incidence of the metabolic syndrome and its constituents in unaffected Taiwanese from 1998 to 2002," Asia Pacific Journal of Clinical Nutrition, vol. 17, no. 2, pp. 339-351, 2008.

[27] W. Zidi, M. Allal-Elasmi, Y. Zayani et al., "Metabolic syndrome, independent predictor for coronary artery disease," Clinical Laboratory, vol. 61, no. 10, pp. 1545-1552, 2015.

[28] J. Lu, L. Wang, M. Li et al., "Metabolic syndrome among adults in China: the 2010 China noncommunicable disease surveillance," The Journal of Clinical Endocrinology and Metabolism, vol. 102, no. 2, pp. 507-515, 2017.

[29] G. A. Jin-li, P. E. Kui, N. I. Heng-ru, H. U. Xiao-lin, B. I. Yufang, and X. U. Min, "Study on the association between metabolic syndrome and risk of cardiovascular disease in middleaged and elderly community-dwelling residents in Shanghai," Journal of Shanghai Jiaotong University, vol. 36, no. 9, pp. 1341-1345, 2016.

[30] A. Ventriglio, A. Gentile, E. Stella, and A. Bellomo, "Metabolic issues in patients affected by schizophrenia: clinical characteristics and medical management," Frontiers in Neuroscience, vol. 9, no. 297, 2015.

[31] Y. Zhu, H. Ji, L. Tao et al., "Functional status of hypothalamicpituitary-thyroid and hypothalamic-pituitary-adrenal axes in hospitalized schizophrenics in Shanghai," Frontiers in Psychiatry, vol. 11, 2020.

[32] V. Soria, A. González-Rodríguez, E. Huerta-Ramos et al., “Targeting hypothalamic-pituitary-adrenal axis hormones and sex steroids for improving cognition in major mood disorders and schizophrenia: a systematic review and narrative synthesis," Psychoneuroendocrinology, vol. 93, pp. 8-19, 2018.

[33] N. Samaras, M. A. Papadopoulou, D. Samaras, and F. Ongaro, "Off-label use of hormones as an antiaging strategy: a review," Clinical Interventions in Aging, vol. 9, pp. 1175-1186, 2014.
[34] B. B. Yeap, L. Manning, S. A. P. Chubb et al., "Progressive impairment of testicular endocrine function in ageing men: testosterone and dihydrotestosterone decrease, and luteinizing hormone increases, in men transitioning from the 8th to 9th decades of life," Clinical Endocrinology, vol. 88, no. 1, article 13484, pp. 88-95, 2018.

[35] N. Manzanares, R. Monseny, L. Ortega et al., "Unhealthy lifestyle in early psychoses: the role of life stress and the hypothalamic-pituitary-adrenal axis," Psychoneuroendocrinology, vol. 39, pp. 1-10, 2014.

[36] B. Konarzewska, B. Galinska-Skok, N. Waszkiewicz, J. Łazarczyk-Kirejczyk, A. Małus, and K. Simonienko, "Association between serum testosterone levels, body mass index (BMI) and insulin in male patients with schizophrenia treated with atypical antipsychotics-olanzapine or risperidone," Neuro Endocrinology Letters, vol. 35, no. 1, pp. 50-57, 2014.

[37] G. Piriu, E. Torac, L. E. Gaman et al., "Clozapine and risperidone influence on cortisol and estradiol levels in male patients with schizophrenia," Journal of Medicine and Life, vol. 8, no. 4, pp. 548-551, 2015.

[38] S. Cohrs, C. Röher, W. Jordan et al., "The atypical antipsychotics olanzapine and quetiapine, but not haloperidol, reduce ACTH and cortisol secretion in healthy subjects," Psychopharmacology, vol. 185, no. 1, pp. 11-18, 2006.

[39] D. Vancampfort, B. Stubbs, A. J. Mitchell et al., "Risk of metabolic syndrome and its components in people with schizophrenia and related psychotic disorders, bipolar disorder and major depressive disorder: a systematic review and meta-analysis," World Psychiatry, vol. 14, no. 3, pp. 339-347, 2015.

[40] A. Tchicaya, N. Lorentz, S. Demarest, J. Beissel, and D. R. Wagner, "Relationship between self-reported weight change, educational status, and health-related quality of life in patients with diabetes in Luxembourg," Health and Quality of Life Outcomes, vol. 13, no. 1, pp. 015-0348, 2015.

[41] A. P. Bress, B. K. Bellows, J. B. King et al., "Cost-effectiveness of intensive versus standard blood-pressure control," The New England Journal of Medicine, vol. 377, no. 8, pp. 745-755, 2017.

[42] Y. Huang, Y. Wang, H. Wang et al., "Prevalence of mental disorders in China: a cross-sectional epidemiological study," Lancet Psychiatry, vol. 6, no. 3, pp. 211-224, 2019. 